Canadian Oncology

Nursing Journal

Revue canadienne

de soins infirmiers

en oncologie

Volume 30, Issue 3 • Summer 2020

elSSN: 2368-8076 


\section{Tournés vers l'avenir : Élaboration du Cadre des connaissances et de la pratique en soins infirmiers contre le cancer}

par Allyson Nowell, Colleen Campbell

RÉSUMÉ

L'Association canadienne des infirmières en oncologie (ACIO/ CANO) est l'organisation nationale qui soutient les infirmières afin de favoriser l'excellence dans la pratique infirmière, l'enseignement, la recherche et le leadership en oncologie. Pour soutenir sa mission, l'ACIO/CANO a elaboré des normes de soins pour les patients atteints de cancer ainsi que des normes et des compétences pour les infirmières en oncologie prenant soin de ces personnes (CANO/ ACIO, 2001, 2006). Depuis la création des premières normes de soins infirmiers spécialisés en oncologie en 2006, les soins contre le cancer ont changé considérablement, notamment avec la multiplication des occurrences et de la prévalence du cancer, lémergence de nouveaux traitements contre le cancer comme les agents par voie orale, de même que la transition des soins contre le cancer hors des centres spécialisés de traitement (CCS/SCC, 2019). Compte tenu du paysage en mutation de la pratique infirmière, l'ACIO/ CANO a entrepris de mettre à jour ses normes dans le but d'y intégrer les infirmières de tous les milieux prenant soin de patients et de familles touchés par le cancer. Dans l'exercice, les spécialistes ont relevé le besoin d'établir un cadre national de soins infirmiers pour mieux intégrer ces mises à jour et brosser un tableau de la contribution infirmière aux soins oncologiques de grande qualité. Le présent article décrit le processus suivi par l'ACIO/CANO pour élaborer le Cadre des connaissances et de la pratique en soins infirmiers contre le cancer.

\section{CONTEXTE}

$\mathbf{I}_{\mathrm{c}}^{\mathrm{c}}$ ci comme ailleurs, le système de lutte contre le cancer se complexifie, et on remarque par ricochet une multiplication des besoins des personnes et familles touchées par le cancer (Bilodeau, Tremblay et Durand, 2019; Saria et al., 2017). Au Canada, ces besoins ne sont pas tous comblés également d'un océan à l'autre (Shakeel, Tung, Rahal et Finley, 2020). Puisque près d'un Canadien sur deux recevra un diagnostic de cancer au cours de sa vie, et que les trois quarts de ce nombre devront

\footnotetext{
AUTEURES

Allyson Nowell, inf. aut., M.Sc.inf., CSIO(C)*

Directrice des services cliniques

Kensington Hospice

38 Major Street, Toronto (Ontario) M5S 2L1

anowell@kensingtonhealth.org

Colleen Campbell, inf. aut. (cat. spéc.), M.Sc.inf., CSIO(C)

Infirmière praticienne en hématologie, cadre infirmière de la région centrale

Centre régional de cancérologie de Southlake

Newmarket (Ontario)

*Auteure-ressource
}

DOI:10.5737/23688076303212216 vivre avec le cancer, les infirmières de bien des milieux devront prodiguer des soins à des patients vivant avec le cancer.

À l'heure actuelle, trop peu de contenu relatif au cancer est enseigné dans les programmes de sciences infirmières, tant au premier cycle qu'aux cycles supérieurs. Devant cette réalité, les infirmières œuvrant dans des centres de traitement contre le cancer enrichissent leurs connaissances sur le domaine au moyen de cours en ligne, de programmes de mentorat formel ou informel et de ressources et soutien de leur organisation nationale d'infirmières en oncologie, l'ACIO/CANO (Charalambouse et Kaite, 2013; International Society of Nurses in Cancer Care, 2013). Or, les infirmières diplômées et celles pratiquant en milieu non oncologique risquent bien, elles, de ne pas avoir toutes les connaissances et compétences essentielles pour s'occuper des patients et familles touchés par le cancer (CNA/AIIC, 2019), d'autant plus que les infirmières pratiquant hors des centres de traitement du cancer ont rarement accès à des formations complémentaires en soins infirmiers spécialisés en oncologie.

La subtilité des traitements du cancer, le recours croissant aux agents par voie orale, l'administration d'une grande partie des traitements en clinique externe et les répercussions multidimensionnelles du cancer dans la trajectoire de soins rendent impératifs l'acquisition de compétences chez les infirmières de tous les milieux de soins, de même que l'intégration dans les programmes de sciences infirmières de tous niveaux (technique collégiale, baccalauréat et études supérieures) de notions sur la complexité des soins infirmiers spécialisés en oncologie (Saria et al., 2017; Wood, 2011, Bonnacchi et al.; 2016, Barsky-Reese et al., 2014; Harrington, Hansen, Moskowitz, et Todd, 2010; Cooley, 2000; Soothill et al., 2002).

A titre d'organisation nationale des infirmières en oncologie, l'ACIO/CANO a fixé, en 2001, des normes de soins centrés sur les patients. Par la suite, en 2006, elle a établi des Normes de pratique et compétences pour l'infirmière spécialisée en oncologie. Ces dernières mettaient principalement l'accent sur la pratique infirmière spécialisée en oncologie. D'autres normes, axées sur la pratique infirmière liée aux soins des patients recevant des types de traitements particuliers (chimiothérapie, radiothérapie, etc.), ont ensuite été produites. Elles encadrent toutes la prestation de soins infirmiers de grande qualité en milieu spécialisé pour le diagnostic et le traitement du cancer (CANO/ACIO, 2015, 2017). Comme la prestation de soins oncologiques n'est plus exclusive aux milieux spécialisés et qu'un accent est maintenant mis aussi sur les phases de prévention et de survie dans le continuum de cancer, les discussions à l'échelle nationale ont fait ressortir 
la nécessité de soutenir et d'orienter les infirmières qui participent aux soins liés au cancer dans tous les milieux.

Les restrictions budgétaires continuant de se conjuguer à une pénurie croissante d'effectifs, il faut absolument que les infirmières de tous les groupes de réglementation exercent pleinement leur pratique (Canadian Nurses Association/ Association des infirmières et infirmiers du Canada [CNA/ AIIC], 2015; Cancer Care Ontario/Action Cancer Ontario [CCO/ACO], 2018). Les infirmières, les pédagogues et les dirigeants des organisations professionnelles de soins infirmiers ont signalé un manque de clarté dans les normes, de même que des lacunes dans les compétences et la formation préalable des infirmières travaillant en dehors des centres spécialisés en oncologie. L'ACIO/CANO, en tant qu'association nationale représentant ces infirmières, a élaboré un cadre visant à orienter les rôles de soins infirmiers dans tous les milieux de pratique afin de garantir l'offre de services de grande qualité qui répondent aux besoins des personnes à risque ou ayant un cancer. Ultimement, si nous unissons nos efforts, nous serons en mesure de réduire l'incidence du cancer au Canada.

\section{ÉLABORATION DU CADRE}

\section{Participation des parties prenantes et des experts}

Dans les Normes de soins rédigées en 2001, l'ACIO/CANO avait classé les rôles infirmiers en trois catégories : infirmières généralistes, spécialisées et en pratique avancée. Les deux dernières catégories étaient décrites en détail. Les soins oncologiques ayant évolué et la prestation des soins ayant basculé en mode communautaire et ambulatoire, le besoin est apparu d'articuler plus en détail le rôle d'infirmière généraliste et les champs de pratique infirmière en soins oncologiques.

En 2015, l’ACIO/CANO a interrogé par téléphone ( $n=$ I9) et en groupes de discussion $(n=42)$ des membres et des infirmières spécialisées en oncologie pour recueillir leur opinion sur la pertinence des normes de soins ainsi que sur les lacunes et enjeux de l'heure liés au traitement du cancer au Canada. Tous s'entendaient pour dire que les Normes de soins et les Normes de pratique et compétences pour l'infirmière spécialisée en oncologie étaient encore pertinentes et suivies dans les établissements de soins spécialisés en oncologie, mais on a par ailleurs signalé des lacunes dans d'autres milieux de pratique relativement aux soins infirmiers prodigués aux patients à risque ou ayant survécu à un cancer après avoir terminé leur traitement. En discutant avec des membres de l'ACIO/CANO, l'équipe chargée du projet a cerné un besoin : celui de soutenir les infirmières soignant des patients atteints du cancer en dehors des établissements spécialisés en oncologie et des centres régionaux de traitement du cancer. Elle a également relevé le besoin d'intégrer dans la nouvelle version des documents les autres rôles infirmiers, comme les infirmières auxiliaires autorisées (inf. aux. aut.) et les infirmières en pratique avancée (y compris les infirmières praticiennes et les infirmières cliniciennes spécialisées).

Après avoir reçu ces commentaires, l'équipe du projet de l'ACIO/CANO a entrepris une étude internationale sur les démarches menées par d'autres organisations infirmières au sujet des rôles infirmiers, des normes et des compétences en oncologie. Léquipe a découvert que peu de documentation décrit la pratique oncologique, en dehors du travail de l'infirmière spécialisée en oncologie. Un cadre rédigé par Cancer Australia présente, pour un modèle de perfectionnement professionnel produit à l'intention des infirmières prodiguant des soins oncologiques, quatre catégories d'infirmières (Aranda et Yates, 2009). Même si ce modèle avait été conçu dans un contexte de formation continue, le cadre fournit une assise utile pour décrire la contribution du personnel infirmier dans les soins oncologiques.

\section{Adaptation du cadre}

Le conseil d'administration de l'ACIO/CANO, piloté par la conseillère générale, pratique professionnelle (auteure AN), a examiné le cadre de perfectionnement professionnel national de l'Australie (EdCaN). Les membres ont réfléchi à la façon d'adapter les éléments pour qu'ils servent de cadre de connaissances et des soins infirmiers dans lequel les normes actuelles de l'ACIO/CANO s'inscriraient (Aranda et Yates, 2009). Les membres du conseil d'administration pensent que l'adoption et l'adaptation du cadre australien EdCaN permettraient de faire progresser davantage le rôle d'infirmière généraliste dans les soins contre le cancer. Parmi les difficultés liées à la création d'un rôle généraliste résidait la nécessité de couvrir divers milieux et besoins en soins (ex. patients en hospitalisation chirurgicale avec une majorité de cas oncologiques versus le milieu communautaire avec quelques patients atteints de cancer et recevant une chimiothérapie).

Une fois que le conseil d'administration a décidé de s'inspirer du cadre australien EdCaN, l'ACIO/CANO a entrepris de consulter ses membres; il s'agissait là d'une étape cruciale pour recueillir l'opinion des spécialistes sur l'applicabilité d'EdCaN au contexte de pratique canadien. Ledit cadre a initialement été présenté lors d'un atelier pendant la conférence de l'ACIO/CANO de 2016. Les 33 participants occupaient des rôles infirmiers en oncologie variés un peu partout au Canada. Pendant la discussion interactive de groupe, les participants ont donné leur avis sur l'élaboration du champ d'application général de la démarche canadienne pour adapter le cadre EdCaN et définir la fourchette de rôles infirmiers en oncologie, de même que les compétences nécessaires à chacun de ces rôles. De manière générale, le cadre EdCaN a été jugé comme une bonne base, que l'on pourrait adapter au contexte canadien. Des différences langagières et de pratiques contenues qui seraient à modifier pour correspondre à l'approche infirmière en oncologie du Canada ont par ailleurs été soulignées.

Ensuite, un groupe spécialisé de travail composé de membres de l'ACIO/CANO de partout au pays a été constitué pour concevoir le cadre canadien. Le groupe avait d'abord pour mission d'établir le but et les objectifs du cadre canadien. Les définitions des différentes catégories de rôles infirmiers ont fait l'objet d'un examen, et des discussions ont été tenues sur la façon dont ces rôles se greffaient aux différents points du continuum de soins en oncologie ainsi que l'endroit où ils s'insèrent dans les quatre domaines du cadre. Ces domaines se basent sur la quantité de patients atteints du cancer avec 
lesquels les infirmières interagissent dans leur milieu de soins. Ils se déclinent ainsi : éléments touchant « toutes les infirmières », « de nombreuses infirmières », « quelques infirmières » et « un petit nombre d'infirmières ».

Une fois le texte, le but et les définitions du cadre canadien rédigés, le résultat a été présenté à un atelier lors de la conférence nationale de l'ACIO/CANO en 2017. Cette étape visait à recueillir les commentaires des membres spécialistes concernant la clarté des documents et à vérifier que le nouveau cadre s'avérerait réellement utile. Trente participants ont indiqué que le cadre serait utile et ont commencé à donner des commentaires sur les normes et les compétences nécessaires au soutien des infirmières œuvrant dans les différents domaines du cadre.

À la suite de cette séance, le nouveau cadre a été soumis à l'examen et l'approbation finale du conseil d'administration de l'ACIO/CANO. Le conseil d'administration a formulé des commentaires et adopté le cadre à l'unanimité (voir la figure 1).

\section{CADRE DES CONNAISSANCES ET DE LA PRATIQUE EN SOINS INFIRMIERS CONTRE LE CANCER DE L'ACPAU}

La présente section donne un aperçu des éléments du nouveau cadre.

\section{But du cadre}

- Encadrer les connaissances et la pratique infirmière pour guider, dans tout le continuum, les soins prodigués aux patients et aux familles touchés par le cancer.
- Indiquer dans quelle catégorie de domaine du cadre se classe une infirmière selon la quantité de personnes atteintes de cancer dont elle prend soin dans son contexte de travail particulier : «toutes les infirmières » concernera un milieu où une partie minime des patients soignés sont atteints du cancer; « beaucoup d'infirmières », un milieu où le nombre de patients atteints du cancer se fait croissant; " plusieurs infirmières », où la majorité des patients soignés sont atteints du cancer; et « quelques infirmières » touchera la pratique infirmière avancée dans un établissement spécialisé en soins oncologiques.

Objectifs du cadre

a) Mettre en évidence la contribution infirmière aux soins oncologiques de grande qualité.

b) Présenter les connaissances infirmières en oncologie requise dans tout le continuum de soins dispensés aux patients atteints du cancer.

c) Énoncer les normes et les compétences nécessaires aux soins infirmiers en oncologie.

d) Aborder la variété des rôles infirmiers dans l'ensemble du spectre des soins oncologiques.

Fondements du cadre :

- Souligner les connaissances en oncologie attendues de toutes les infirmières, où qu'elles travaillent.

- Veiller à ce que les soins prodigués aux patients atteints du cancer soient de grande qualité.

- Insister sur la collaboration essentielle de toutes les infirmières dans l'ensemble du continuum.

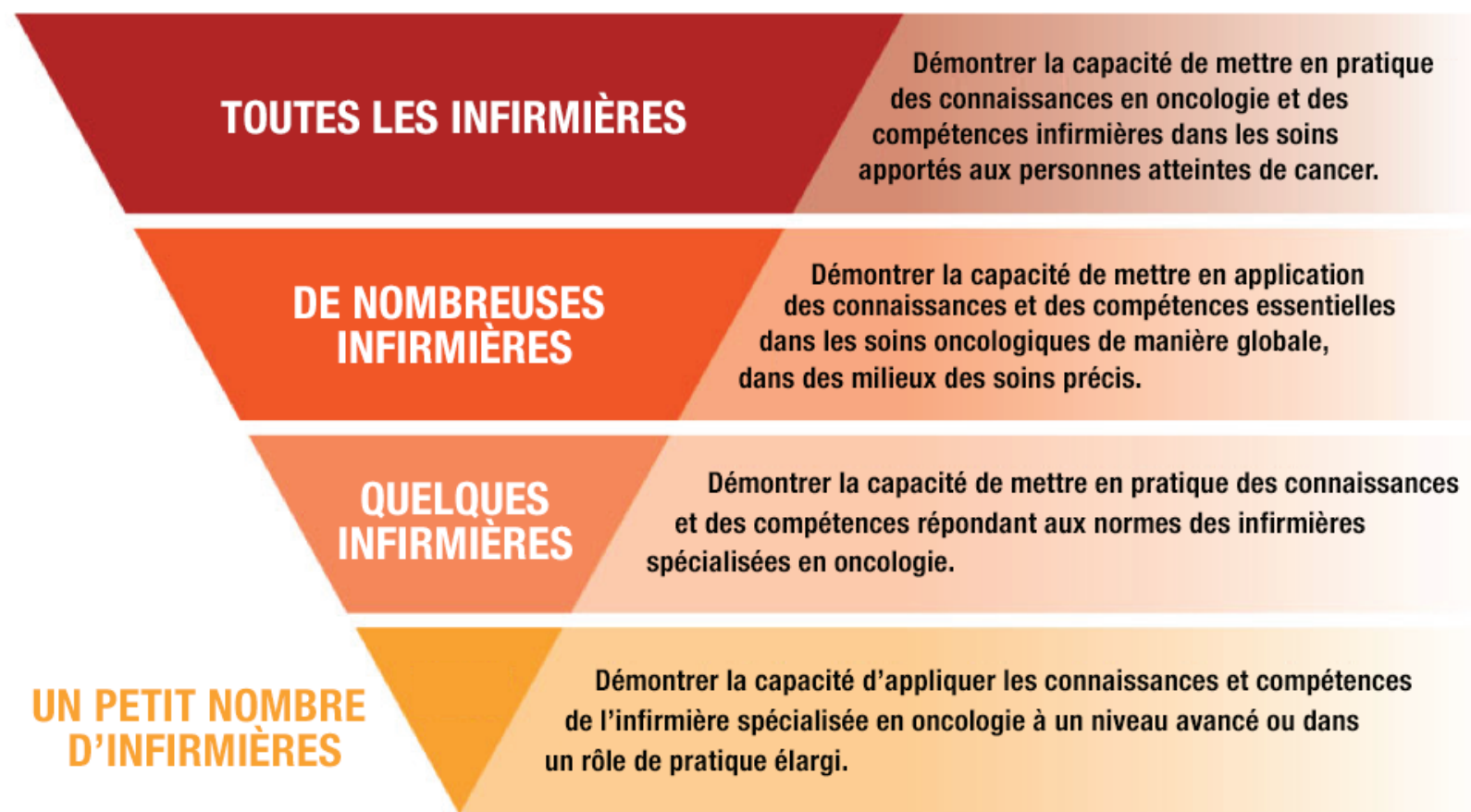

Figure 1. Définitions du cadre 


\section{CADRE DES COMPÉTENCES ET CONNAISSANCES INFIRMIĖRES POUR LES SOINS ONCOLOGIQUES DE L'ACPAU}

\section{Création de la boîte à outils du cadre}

À la suite du lancement du Cadre des connaissances et de la pratique en soins infirmiers contre le cancer de l'ACIO/CANO, le comité de la pratique professionnelle de l'ACIO/CANO a déterminé qu'il était nécessaire de mettre en place des ressources afin de favoriser l'adhésion au cadre dans l'ensemble des milieux de soins. Lors de l'atelier de 2017, les participants ont confirmé que les Normes et compétences en matière de pratique de soins infirmiers spécialisés en oncologie de 2006 s'appliquaient à la pratique infirmière et pouvaient être adaptés pour représenter les quatre domaines du cadre. Le comité a alors entrepris de rédiger un document d'accompagnement et une boîte à outils décrivant le besoin pour le cadre dans les milieux de soins, en abordant tous les rôles infirmiers qui prennent soin de personnes atteintes de cancer et en établissant des normes de pratiques rigoureuses. Des situations cliniques y ont également été décrites afin d'assurer une bonne compréhension des soins oncologiques dans tous les milieux.

Pendant le processus de rédaction du document final et de la boîte à outils, des membres du Comité de la pratique professionnelle de l'ACIO/CANO, à titre de spécialistes en soins infirmiers en oncologie, ont examiné les normes en vigueur et ont déterminé la façon dont elles seraient appliquées à chaque domaine du cadre afin de cultiver les compétences requises. Chaque norme était finalisée par une dyade, puis revue et peaufinée avec tout le groupe. Pour vérifier la pertinence et l'applicabilité du document en milieux non spécialisés en oncologie, un sondage a été mené auprès des intervenants. Il a été distribué à des infirmières œuvrant en milieu non oncologique. Nous avons reçu 18 réponses, et la grande majorité (94\%) des répondants ont jugé que le document s'appliquait à leur milieu, jugeant qu'il témoignait bien de l'étendue des connaissances liées aux soins du cancer que doivent posséder les infirmières dans les établissements d'oncologie non spécialisés.

Le document, intitulé Cadre des connaissances et de la pratique en soins infirmiers contre le cancer, souligne les connaissances oncologiques attendues pour toutes les infirmières, indépendamment de leur milieu de travail et constitue un outil au service des infirmières, des dirigeants, des pédagogues et des décideurs politiques.

\section{Résumé}

Toutes les infirmières travaillant auprès de patients et familles touchés par le cancer, indépendamment de leur milieu ou de leur réglementation, doivent détenir des connaissances fondamentales en oncologie. Comme le nombre de personnes souffrant du cancer en même temps que le nombre de modalités de traitement dispensées à l'externe s'accroît, toutes les infirmières, quel que soit leur milieu de travail, pourraient être amenées à prendre soin de patients et de familles touchés par le cancer, y compris en soin longue durée, en soins primaires et en unités pour patients hospitalisés. Consciente des lacunes sur le plan de l'éducation et du besoin de soutenir les soins infirmiers en oncologie de grande qualité, l'ACIO/CANO a créé les Normes de pratique et compétences pour l'infirmière spécialisée en oncologie (2006). Toutefois, nombreuses sont les infirmières hors des établissements oncologiques qui ne connaissent pas les normes ou la façon de les adapter aux milieux non oncologiques. Voyant le besoin de soutenir ces infirmières, l'ACIO/ CANO a adopté le Cadre des connaissances pour les soins contre le cancer. Pour favoriser l'application des connaissances du cadre, une boîte à outils décrivant comment les normes et les compétences attendues s'inscrivent dans le cadre a été élaborée.

\section{CONCLUSION}

En raison du nombre croissant de patients atteints du cancer et de survivants du cancer dans la population canadienne, les infirmières travaillant dans n'importe quel milieu sont désormais susceptibles d'interagir avec des patients et des familles à diverses étapes dans le continuum de l'expérience du cancer. Les infirmières œuvrant dans des milieux de traitement spécialisés reçoivent $d u$ soutien et des ressources pour se perfectionner et parfaire leurs compétences, mais la vaste majorité des infirmières travaillant dans les hôpitaux ou en milieu communautaire n'ont pas cette formation ou des normes de pratique pour encadrer les soins oncologiques et soutenir leur pratique professionnelle de soin des personnes atteintes de cancer. Le Cadre des connaissances et de la pratique en soins infirmiers contre le cancer de l'ACIO/CANO décrit la contribution infirmière aux soins oncologiques de grande qualité, reconnaît les connaissances infirmières nécessaires pour dispenser des soins et soutient tous les rôles infirmiers d'un bout à l'autre du continuum de soins oncologiques. Une boîte à outils soutenant l'application des connaissances liées au cadre a été créée et intègre les normes pour les quatre domaines énoncés dans le cadre. Cette ressource vise à fournir une excellente base pour préparer les infirmières et soutenir leur pratique dans les soins offerts aux patients atteints de cancer, indépendamment de leur milieu de pratique ou de leur formation.

\section{REMERCIEMENTS}

Les auteures souhaitent remercier tous les membres du Comité de la pratique professionnelle pour le travail mis dans la préparation de la boîte à outils du cadre, de même que tous les spécialistes des soins infirmiers en oncologie qui ont donné leur avis pendant ces travaux. 


\section{RÉFÉRENCES}

Aranda, S., \& Yates, P. (2009). A national professional development framework for cancer nursing. (2nd ed.). The National Cancer Nursing Education Project (EdCaN). https://www.Edcan.org.au/ professional-development/edcan-framework

Barsky Reese, J., Porter, L., Regan, K., Keefe, F., Azad, N., Diaz, L., Herman, J., \& Haythornthwaite, J. (2014). A randomized pilot trial of a telephone-based couples intervention for physical intimacy and sexual concerns in colorectal cancer. Psycho-oncology, 23(9), 1005-1013. https://doi.org/10.1002/pon.3508

Bilodeau, K., Tremblay, D. \& Durand, M.J. (2019). Gaps and delays in survivorship care in the return-to-work pathway for survivors of breast cancer-A qualitative study. Current Oncology, 26(3), http:// dx.doi.org/10.3747/co.26.4787

Bonacchi, A., Miccinesi, G., Galli, S., Primi, C., Chiesi, F., Lippi, D., Muraca, M., \& Toccafondi, A. (2016). Use of the Needs Evaluation Questionnaire with cancer outpatients. Supportive Care in Cancer, 24(8), 3507-3515.

Canadian Association of Nurses in Oncology. (2006). Practice Standards and Competencies for the Specialized Oncology Nurse. https://cdn.ymaws.com/www.cano-acio.ca/resource/resmgr/ standards/CONEP_Standards2006September.pdf

Canadian Association of Nurses in Oncology (2001). Standards of Care. https://cdn.ymaws.com/www.cano-acio.ca/resource/resmgr/ standards/Standards_of_Care_ENG.pdf

Canadian Association of Nurses in Oncology (2017). Standards and Competencies for Cancer Chemotherapy Nursing Practice. https://cdn. ymaws.com/www.cano-acio.ca/resource/resmgr/Resources/EN_ CANO_Chemotherapy_Standar.pdf

Canadian Association of Nurses in Oncology (2015). Radiation Oncology Nursing Practice Standards and Competencies. https:// cdn.ymaws.com/www.cano-acio.ca/resource/resmgr/standards/ ronp_s\&c_web(2).pdf

Canadian Cancer Society (2019). Cancer Statistics at a glance. http://www.cancer.ca/en/cancer-information/cancer-101/ cancer-statistics-at-a-glance/?region=on

Canadian Nurses Association. (2015). Framework for the Practice of Registered Nurses in Canada. https://www.cna-aiic.ca/-/media/cna/ page-content/pdf-en/framework-for-the-pracice-of-registerednurses-in-canada.pdf
Canadian Nurses Association. (2019). Advanced Practice Nursing, A Pan-Canadian Framework. Canadian Nurses Association: $\quad$ https://cna-aiic.ca/-/media/cna/page-content/ pdf-en/apn-a-pan-canadian-framework.pdf?la=en \&hash= E1387634D492FD2B003964E3CD4188971305469E

Cancer Care Ontario: Ontario Oncology Advanced Practice Nursing Community of Practice (2018). Expert recommendations report on clinical nurse specialist and nurse practitioner roles in the delivery of adult cancer services in Ontario. https://www.cancercareontario.ca/ en/guidelines-advice/types-of-cancer/52201

Charalambouse, A., \& Kaite, C., (2013). Undergraduate nursing students caring for cancer patients: hermeneutic phenomenological insights of their experiences. BMC Health Services, 13(63). https://doi.org/10.1186/1472-6963-13-63

Cooley, M. (2000). Symptoms in adults with lung cancer. Journal of Pain and Symptom Management, 19(2), 137-153.

Harrington, C. B. C., Hansen, J. A., Moskowitz, M., \& Todd B. L. (2010). It's not over when it's over: Long-term symptoms in cancer survivors: A systematic review. International Journal of Psychiatry in Medicine, 40(2), 163-181.

International Society of Cancer Nursing. (2013). Cancer Nursing Education Position Statement. https://cdn.ymaws.com/www.isncc. org/resource/resmgr/Position_Statements/ISNCC_Cancer_ Nursing_Educati.pdf

Saria, M., Nyamathi, A., Phillips, L., Stanton, A., Evangelista, L., Kesari, S., \& Maliski, S. (2017). The hidden morbidity of cancer: Burden in caregivers of patients with brain metastases. The Nursing Clinics of North America, 52(1), 159-178.

Shakeel, S., Tung, J., Rahal, R., Finley, C. (2020). Evaluation of Factors Associated with Unmet Needs in Adult Cancer Survivors in Canada. JAMA Netw Open, 3(3), e200506. https://doi.org/10.1001/ jamanetworkopen.2020.0506

Soothill, K., Morris, S. M., Harman, J. C., Francis, B., Thomas, C., \& Mcillmurray, M. B. (2002). Informal carers of cancer patients: What are their unmet needs? Health and Social Care, 9(6), 476-489.

Wood, L. (2011). A review on adherence management in patients on oral cancer therapies. European Journal of Oncology Nursing, 16(4), $432-438$. 